\title{
Mechanical support of total cavopulmonary connection with an axial flow pump
}

\author{
R. Kirk Riemer, $\mathrm{PhD},{ }^{a}$ Gabriel Amir, $\mathrm{MD}^{\text {a }}$ Steven H. Reichenbach, $\mathrm{PhD}{ }^{\mathrm{b}}$ and Olaf Reinhartz, $\mathrm{MD}^{\mathrm{a}}$
}

From the Department of Cardiothoracic Surgery, ${ }^{\mathrm{a}}$ Stanford University, Stanford, Calif, and the Department of Research and Development, ${ }^{\text {b }}$ Thoratec Inc, Pleasanton, Calif.

This project is supported by the American Heart Association Western States Affiliate grant 0365113y (Reinhartz). W. L. Gore \& Associates, Inc, provided the Gore-Tex graft material used in these studies. Dr Amir is the recipient of a fellowship from the American Physician Fellowship for Medicine in Israel.

Presented in part at the 50th annual ASAIO Conference, Washington, DC, June 2004.

Received for publication Sept 10, 2004; revisions received Dec 20, 2004; accepted for publication Dec 29, 2004.

Address for reprints: Olaf Reinhartz, MD, Department of Cardiothoracic Surgery, Stanford University, Stanford, CA 943055407 (E-mail: orx@stanford.edu).

J Thorac Cardiovasc Surg 2005;130:351-4

$0022-5223 / \$ 30.00$

Copyright $(9) 2005$ by The American Association for Thoracic Surgery

doi:10.1016/j.jtcvs.2004.12.037
Objective: Even under optimal circumstances, total cavopulmonary connection is associated with a continuous late risk of death. Hemodynamics are distinctly abnormal, with increased systemic venous pressures and frequent low cardiac output. Our study uses a sheep model of total cavopulmonary connection to test the response to axial flow pump (Thoratec HeartMate II; Thoratec Corporation (Pleasanton, Calif) support of total cavopulmonary connection, which might be suitable to treat patients with failing Fontan circulation.

Methods: Eight sheep (42-48 kg) were studied. After pilot studies in 3 animals, 5 underwent both pump-supported and nonsupported total cavopulmonary connection in alternating sequence for up to 2 hours. This was achieved with a $12-\mathrm{mm}$ polytetrafluoroethylene graft from the (distally ligated) superior vena cava to the main pulmonary artery and a cannula placed in the inferior vena cava with an attached 16-mm Dacron graft to the main pulmonary artery. Pressures (arterial, inferior vena cava, left atrium, and pulmonary artery) and flows (ascending aorta and inferior vena cava) were recorded over 1 hour both with unsupported total cavopulmonary connection and after placing an axial flow pump (Thoratec HeartMate II) between the inferior vena caval inflow cannula and the main pulmonary artery.

Results: Under nonsupported total cavopulmonary connection circulation, inferior vena caval and aortic blood flow decreased by nearly 50\%. Inferior vena caval pressure nearly doubled, whereas arterial pressure decreased by one third. Pulmonary artery pressure became nonpulsatile; however, mean pulmonary artery pressure and left atrial pressure did not change significantly. With pump-supported Fontan circulation, cardiac output, inferior vena caval flow, and arterial pressure returned to baseline. Inferior vena caval pressure decreased to below baseline levels. Mean pulmonary artery pressure and left atrial pressure again remained unchanged.

Conclusions: Axial flow pump support from the inferior vena cava to the pulmonary artery can prevent the substantial decrease of aortic flow and pressure associated with total cavopulmonary connection and can reverse its poor hemodynamics. This is a simple model that can be used to further evaluate the potential of mechanical support as a treatment option in failing Fontan circulation.

$\mathrm{T}$ Total cavopulmonary connection (TCPC; Fontan circulation) produces a unique physiology in which pulmonary blood flow is driven primarily by the residual force of the systemic ventricle. It is therefore usually accompanied by substantial increases of systemic venous pressure. Even though most patients' clinical state is good, objectively measured hemodynamics and exercise tolerance are frequently abnormal. Furthermore, there appears to be a continuous late risk of death, even increasing after 6 years postoperatively while functional status decreases. ${ }^{1}$

Up to $40 \%$ of patients undergoing TCPC experience heart failure. ${ }^{2}$ Late abnormalities of liver function are frequent. ${ }^{3}$ Protein-losing enteropathy is diagnosed in 


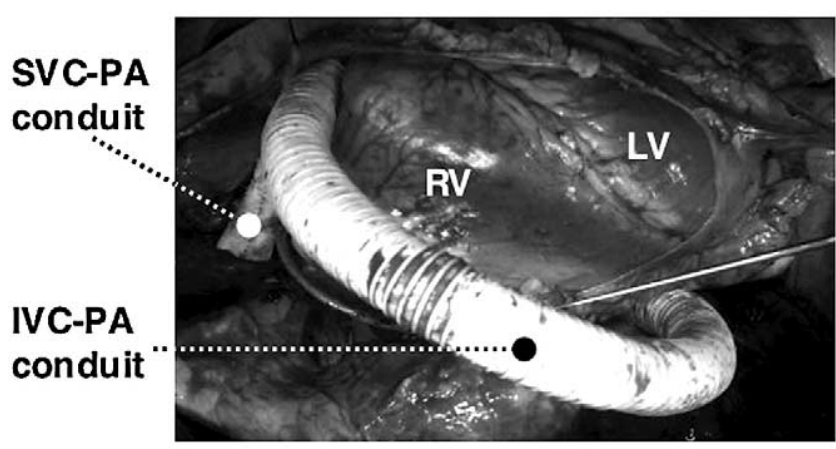

Figure 1. Photograph of TCPC without pump assist in a sheep: surgeon's view showing the SVC-PA and PA-IVC conduits in situ. Portions of the right $(R V)$ and left ventricular $(L V)$ free walls are shown.

up to $15 \%$ of patients and has a 5-year mortality rate of $50 \%{ }^{4}$ Medical treatment options are limited. In rare cases of Fontan pathway obstruction, revision is indicated. Cardiac transplantation is the only remaining option for patients refractory to all other treatments. This option is, however, associated with increased risk because of the poor condition of patients with failing Fontan circulation. ${ }^{5}$

Mechanical circulatory support for left ventricular failure with pulsatile and, more recently, axial flow pumps is now state of the art clinically. However there is little experience with axial flow pumps in low-pressure circuits, such as support of the right ventricle or even a TCPC circulation. Pump support could conceivably reverse poor hemodynamics and secondary organ failure in patients with failing Fontan circulation, could make these patients more successful transplant candidates, or could be used as destination therapy. In this study we compared the acute physiologic response to passive TCPC with that of mechanically supported TCPC by using an axial flow blood pump in a sheep model. Our objective was to define the initial adaptive response of the circulation to passive TCPC and determine the feasibility of mechanical support to restore hemodynamics.

\section{Methods}

Animal Care

We used a total of 8 sheep (Western breed, $42-48 \mathrm{~kg}$ ) in this study; 3 animals were used for preliminary studies. All animals received humane care in compliance with the "Guide for Care and Use of Laboratory Animals." All conditions for animal surgery and care were approved by the Stanford University Institutional Animal Care and Use Committee. Animals were premedicated with ketamine and diazepam (Valium; 10 and $0.5 \mathrm{mg} / \mathrm{kg}$ administered intravenously, respectively), intubated, ventilated to maintain a blood $\mathrm{PCO}_{2}$ value of between 35 and $45 \mathrm{~mm} \mathrm{Hg}$, and maintained under $1 \%$ to $2 \%$ isoflurane anesthesia. At the end of the study, they were killed with pentobarbital $(150 \mathrm{mg} / \mathrm{kg})$.

\section{Surgical Preparation}

We performed a midline sternotomy; inserted pressure catheters into the left atrium, right atrium (RA), inferior vena cava (IVC), and main pulmonary artery (PA); and placed perivascular ultrasonic flow probes (Transonics Systems, Inc, Ithaca, NY) around the IVC distal to the anastomosis and on the ascending aorta. All pressures and flows were continuously monitored and recorded.

We mobilized the IVC, superior vena cava (SVC), and main PA and ligated the azygous vein. After heparinization with 300 $\mathrm{U} / \mathrm{kg}$, we fashioned a bidirectional Glenn connection by anastomosing a 12-mm polytetrafluoroethylene (PTFE; Gore-Tex vascular graft, registered trademark of W. L. Gore \& Associates, Inc, Newark, Del) vascular graft from the SVC to the main PA in side-to-side fashion and ligating the SVC-RA junction. For nonsupported TCPC, we connected the IVC and main PA with a 16-mm PTFE graft and ligated the IVC-RA connection (Figure 1). To test pump-supported TCPC, we placed a right-angled Thoratec inflow cannula connected to an axial flow pump (Thoratec HeartMate II; Thoratec Corporation, Pleasanton, Calif) into the IVC. Its outflow end was connected to a 16-mm Dacron graft anastomosed to the proximal main PA. We then again ligated the IVC-RA connection (Figure 2). These graft diameters were chosen to match the sheep's SVC and IVC diameters. The chest was approximated with clamps. All animals were studied with and without mechanical support in random order. The PTFE graft was clamped before insertion of the axial flow pump, or vice versa, the pump was removed before opening the PTFE graft. Therefore, all animals served as their own controls. Hemodynamic variables were re-

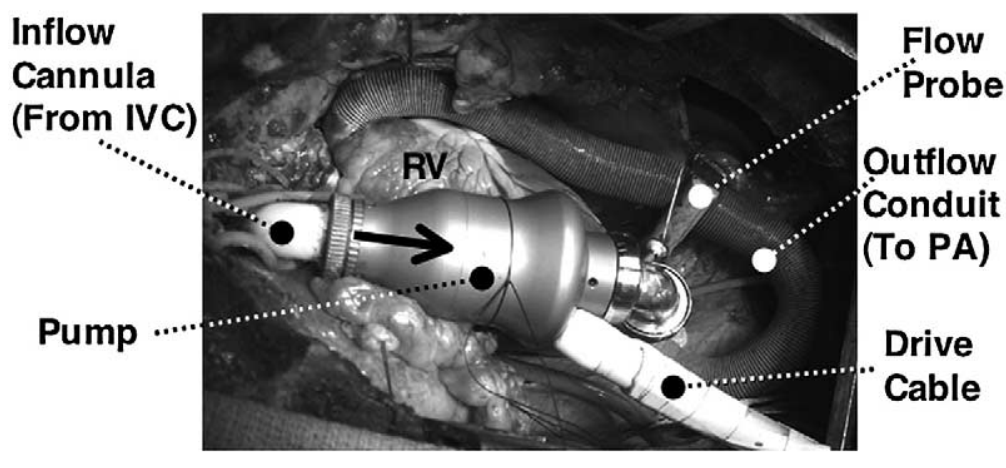

Figure 2. Photograph of TCPC with the HeartMate II ventricular assist device in a sheep: surgeon's view of pump and conduits in situ. Arrow indicates direction of blood flow through the pump. RV, Right ventricular free wall. 
TABLE 1. Hemodynamic values For different circulatory configurations*

\begin{tabular}{lccr}
\hline Circulation & Baseline & TCPC & TCPC with pump \\
\hline IVC flow, L/min & $2.02 \pm 0.21(5)$ & $0.88 \pm 0.10(5) \dagger$ & $2.6 \pm 0.26(4) \ddagger$ \\
Aortic flow (CO), L/min & $3.89 \pm 0.13(5)$ & $2.10 \pm 0.13(5) \dagger$ & $4.18 \pm 0.36(4) \ddagger$ \\
Arterial pressure, mm Hg & $68.9 \pm 3.3(5)$ & $42.4 \pm 42.8(5) \dagger$ & $64.5 \pm 9.13(4) \ddagger$ \\
LA pressure, mm Hg & $6.4 \pm 0.75(5)$ & $5.2 \pm 0.73(5)$ & $7.5 \pm 1.2(4)$ \\
IVC pressure, mm Hg & $5.00 \pm 0.84(5)$ & $10.6 \pm 1.12(5) \dagger$ & $-0.25 \pm 0.48(4) \dagger \ddagger$ \\
PA pressure, mm Hg & $11.8 \pm 0.80(5)$ & $10.0 \pm 1.10(5)$ & $13.75 \pm 1.03(4)$ \\
\hline
\end{tabular}

$T C P C$, Total cavopulmonary connection; $I V C$, inferior vena cava; $C O$, cardiac output; $L A$, left atrial; $P A$, pulmonary artery. *Values are presented as means \pm standard error of the mean (n). $\dagger P<.05$ versus baseline. $\ddagger P<.05$ versus total cavopulmonary connection.

corded over a period of 1 to 2 hours each before intervention and with unsupported and supported TCPC, respectively.

\section{Pump Regulation}

In all animals the pump was set at a speed of $6000 \mathrm{rpm}$ for the duration of pump support. In 3 animals pump speed was varied for a short time to observe changes in hemodynamics and to monitor for IVC collapse. The pump dynamics data will be presented elsewhere.

\section{Metabolic Analyses}

Hourly arterial blood gas measurements were obtained to monitor ventilation and base excess. In addition, oxygen saturations in PA and IVC blood, serum lactate levels, and aspartate aminotransferase and alanine aminotransferase concentrations were measured before and during supported and nonsupported TCPC after 30minute equilibration periods.

\section{Statistical Analysis}

Hemodynamic values were analyzed by means of analysis of variance with paired comparison between baseline, unassisted, and ventricular assist device-assisted states. The analysis was conducted with the JMP software package (SAS Institute, Inc, Cary, NC). A significance level of .05 was the minimum criterion for acceptance of differences between groups. All data values are expressed as means \pm standard error unless otherwise stated.

\section{Results}

\section{Hemodynamics at Baseline and After TCPC}

All animals survived the study. Measured hemodynamic variables are summarized in Table 1. Compared with biventricular circulation, aortic and IVC flow decreased significantly by $56 \% \pm 4 \%$ and $46 \% \pm 4 \%$, respectively. Arterial pressure also decreased significantly with TCPC, whereas left atrial pressure did not change substantially. TCPC did not significantly affect pulmonary arterial pressure. Direct connection with the PA resulted in an IVC pressure increase from baseline (by $130 \% \pm 30 \%$ ) to match PA pressure.

\section{Hemodynamics With Ventricular Assist Device-Supported TCPC}

Axial pump support from the IVC to the PA restored aortic and IVC flows to pre-TCPC values. Arterial pressure also recovered to baseline. IVC pressure was decreased to around $0 \mathrm{~mm} \mathrm{Hg}$, which was significantly less than baseline values. Left atrial pressure again remained unchanged, whereas PA pressure was mildly increased with pump support (Table 1).

\section{Venous Collapse}

At a pump speed of $6000 \mathrm{rpm}$, the IVC was subject to intermittent collapse and oscillation in all animals. However, this did not appear to affect overall IVC flows. In preliminary studies we verified that further increasing pump speed induced more prolonged IVC collapse, leading to brief and intermittent decreases in flow.

\section{Metabolic Changes}

During the relatively brief course of the experiment, we did not observe significant metabolic or lactic acidosis or increases of aspartate aminotransferase or alanine aminotransferase concentrations. Mixed venous saturations were measured in 5 animals: the median was $87 \%$ at baseline, $61 \%$ at the passive Fontan state, and 82\% during Fontan circulation with pump support. Because of the small number of observations, we did not perform any statistical analyses for mixed venous saturations.

\section{Discussion \\ Model of TCPC}

We used a simple and reliable model for TCPC that has not been used in sheep before. This model does not require cardiopulmonary bypass (CPB), avoiding its associated potential volume shifts, blood transfusions, and influences on pulmonary vascular resistance and lung function. It appears well suited to study hemodynamics in Fontan circulation. Two groups have applied a similar model in dogs before: Nawa and colleagues, ${ }^{6}$ who also avoided CPB, and Mace and associates, ${ }^{7}$ who used CPB. Both were able to show that dogs tolerate TCPC in the acute setting.

A limitation of this model is the incomplete bypass of the right heart in that coronary sinus flow is still being pumped by the right ventricle. However, this did not lead to any appreciable pulsatility of the PA pressure tracing in our study. It appears that coronary sinus flow is not substantial enough to affect the hemodynamics of this experiment significantly. 


\section{Hemodynamics in TCPC}

In the previously reported studies, cardiac index was substantially reduced with TCPC; however, output could be maintained by substantial volume loading. ${ }^{6,7}$ In our sheep model we have seen an even greater decrease of cardiac output and blood pressure with TCPC without volume loading. As expected, IVC pressure was significantly increased in TCPC. Additionally, we measured IVC flow, which was significantly reduced along with cardiac output.

As in the previous studies in dogs, all animals survived in the acute setting. Chronic studies (already underway) will have to be performed to determine whether sheep can survive TCPC long term.

\section{Pump Support of TCPC}

Axial flow pump support from the IVC to the PA reversed the negative hemodynamics of passive TCPC. Cardiac output, IVC flow, and arterial pressure were similar to biventricular circulation without volume administration, illustrating the potential feasibility of pump support of Fontan circulation. IVC pressure in pump-supported TCPC is low, potentially allowing for recovery from liver failure or proteinlosing enteropathy.

Rodefeld and coworkers ${ }^{8}$ were the first to apply pump support of TCPC in an animal model, using 2 Hemopumps in the SVC and IVC. They were able to show feasibility of mechanical support; however, they failed to provide a control group for comparison of hemodynamics. This study uses each animal as its own control, clearly proving the improvement of hemodynamics of pump-supported versus passive Fontan circulation.

\section{IVC Collapse}

The Thoratec HeartMate II is a powerful axial flow pump designed for use in the systemic circulation of adults. We would have considered using a smaller pump in this experiment if it had been available to us. However, despite negative IVC pressures, we did not encounter as many problems with IVC collapse and associated reduction of pump inflow as we anticipated. Even though we consistently experienced oscillations of the IVC wall and moderate IVC collapse, pump flow remained stable throughout at the recommended minimum of $6000 \mathrm{rpm}$. Even with increased revolutions per minute, there was only intermittent and self-limiting inflow reduction.

\section{Thrombosis}

During the short duration of this study in heparinized animals, we did not encounter any thromboembolic problems. This, however, remains a concern, particularly when using a pump designed for higher flows. The need for anticoagulation will have to be evaluated in subsequent chronic studies.

\section{Other Limitations}

Anesthetic drugs are known to adversely affect the hemodynamics of TCPC. Therefore the concentration of inhaled anesthesia was kept constant during the periods of passive and pump-supported TCPC, minimizing its influence on the data.

Positive pressure ventilation could also affect hemodynamics unfavorably. However, in our experience chest approximation did not change pressures and flows significantly. The data in these series of experiments did not suggest major compression of the grafts by means of chest approximation, although we have seen graft compression in chronic preparations when the animal's position was changed.

The relatively large size of the Thoratec HeartMate II pump could obviously be of concern in smaller animals and, eventually, in small patients. It was not a limitation in this study with large sheep.

\section{Conclusions}

Mechanical support of TCPC with an axial flow pump is feasible in the acute experiment in sheep, leading to reversal of the reduction of cardiac output, IVC flow, and blood pressure that is associated with acute TCPC. This principle could lead to further advanced approaches to improve patients with failing Fontan circulation, whose therapeutic options are otherwise severely limited.

We thank Mario Trujillo for technical assistance with the animals.

\section{References}

1. Fontan F, Kirklin JW, Fernandez G, Costa F, Naftel DC, Tritto F, et al. Outcome after a perfect Fontan operation. Circulation. 1990;81: 1520-36.

2. Piran S, Veldtman G, Siu S, Webb GD, Liu PP. Heart failure and ventricular dysfunction in patients with single or systemic right ventricles. Circulation. 2002;105:1189-94.

3. van Nieuwenhuizen RC, Peters M, Lubbers LJ, Trip MD, Tijssen JG, Mulder BJ. Abnormalities in liver function and coagulation profile following the Fontan procedure. Heart. 1999;82:40-6.

4. Rychik J, Spray TL. Strategies to treat protein-losing enteropathy. Semin Thorac Cardiovasc Surg Pediatr Card Surg Annu. 2002;5:3-11.

5. Mitropoulos FA, Laks H, Neelankavil J, Odim J, Alejos J, Kobashigawa $\mathrm{J}$, et al. Orthotopic heart transplantation in patients with failing Fontan circulation. In: 40th Annual Meeting of the Society of Thoracic Surgeons. San Antonio (TX): Society of Thoracic Surgeons; 2004. p. 208.

6. Nawa S, Irie H, Takata K, Sugawara E, Teramoto S. Development of a new experimental model for total exclusion of the right heart without the aid of cardiopulmonary bypass. J Thorac Cardiovasc Surg. 1989; 97:130-4.

7. Mace L, Dervanian P, Weiss M, Daniel JP, Losay J, Neveux JY. Hemodynamics of different degrees of right heart bypass: experimental assessment. Ann Thorac Surg. 1995;60:1230-7.

8. Rodefeld MD, Boyd JH, Myers CD, LaLone BJ, Bezruczko AJ, Potter AW, et al. Cavopulmonary assist: circulatory support for the univentricular Fontan circulation. Ann Thorac Surg. 2003;76: 1911-6. 